\title{
LE PEN ET LE PEUPLE \\ Géopolitiques du vote FN en Languedoc-Roussillon
}

Emmanuel Négrier

\begin{abstract}
Résumé
A l'occasion des élections présidentielles de 2012, la candidature de Marine Le Pen a dépassé de près de 6 points, en Languedoc-Roussillon, une moyenne nationale que beaucoup ont analysée comme un succès pour le Front National. L'évolution de son discours fait la part belle à l'hypothèse d'un renouvellement du parti, qui se revendique d'être désormais l'expression d'un "peuple qui s'invite à la table des élites ». Les approches géographiques insistent, quant à elles, sur un vote qui serait la traduction inéluctable d'un malaise périurbain. Dans ce papier, nous discutons ce premier schéma en nous penchant sur les géographies électorales du Front National. Puis nous discutons l'idée que ce même vote serait l'expression désormais dominante des dominés. La première partie analyse les zones de force du vote FN et ce qu'elles nous disent de ses dynamiques régionales. La seconde partie concerne les caractéristiques du vote dans les zones les plus populaires de la région.
\end{abstract}

Mots-clefs : Front National, Languedoc-Roussillon, Élections Présidentielles, quartiers populaires, espace périurbain et vote

\section{Le Pen and the People. Geopolitics of FN vote in Languedoc-Roussillon Region}

\begin{abstract}
In the French presidential elections of 2012, Marine Le Pen has reached 23,5\% of votes in Languedoc-Roussillon region, nearly 6 points higher than her national average, however regarded as a success for the National Front. The evolution of FN discourse underlines the hypothesis of a renewal of the party, which now claims to be the expression of "the people invited at elites' banquet". Geographical approaches insist, in turn, on this vote as the expression of the "suburban malaise". In this paper, we first discuss this approach by addressing the electoral geography of the National Front in one of its main territories of influence. Then we discuss the idea that this electoral behavior would have become the dominant expression of dominated people. The first part focuses on areas of strength of the FN vote and what they tell us about its regional dynamics. The second part concerns the characteristics of the vote in the most popular areas of the region. Instead of beeing their electoral bastions, popular and dominated neighbourhoods are, more than others, résistant to the FN political stream.
\end{abstract}

Keywords : Front National, Languedoc-Roussillon, Presidential Election, popular neighbourhoods, suburbanism and politics. 


\section{Introduction}

Le Languedoc-Roussillon s'est à nouveau illustré comme l'une des régions clefs du vote $\mathrm{FN}^{1}$, tant par l'ampleur des suffrages obtenus par Marine Le Pen $(23,45 \%)$ que par le fait que l'un de ses départements, le Gard, a été le seul à placer la présidente du FN en tête de tous les candidats, sans pour autant établir de record départemental pour le FN : aux 25,51\%, le Gard, on peut opposer les 25,53\% dans le Pas-de-Calais, 25,82\% dans la Meuse et même $26,33 \%$ dans l'Aisne. Surtout, ce score a été obtenu dans un contexte de participation assez élevée, ce qui tranche avec l'élection présidentielle la plus comparable, celle de 2002. L'extrême-droite, se présentant alors divisée (Jean-Marie Le Pen et Bruno Mégret), avait obtenu un meilleur pourcentage $(24,92 \%$, soit $1,5 \%$ de mieux qu'en 2012), mais 65000 voix de moins. Dans les deux cas, la sur-représentation qu'elle connaît en Languedoc-Roussillon par rapport à sa moyenne nationale est la même : $+5,55 \%$.

Les élections présidentielles et législatives 2012 se déroulent, en Languedoc-Roussillon, dans un double contexte. D'une part, c'est le malaise lié à des relations conflictuelles avec la direction nationale du parti, plus d'un an après la disparition de son leader régional, Georges Frêche, qui n'avait pas été pour rien dans ces conflits. D'autre part, la gauche exerce une très forte domination à l'échelle des collectivités territoriales, illustrant jusqu'à l'extrême les analyses critiques sur la territorialisation du PS (Lefebvre \& Sawicki 2006). Elle truste, depuis 1998, plus de la moitié des villes de plus de 3500 habitants, dont la capitale Montpellier, 4 des 5 conseils généraux, la Lozère, seule, lui échappant. La gauche gouverne également la Région depuis 2004. C'est à l'Assemblée nationale qu'elle est plus en difficulté, en ne comptant, avant juin 2012, que 6 députés sur 21. Au Sénat, en revanche, 8 représentants sur 12 sont socialistes ou apparentés.

Dans ce contexte, on pouvait imaginer que la gauche allait réserver à ses candidats les scores les plus élevés, avec, comme nous l'avons souvent remarqué à l'occasion des précédentes élections, une participation également plus forte. Si celle-ci est confirmée, avec près de $3 \%$ de plus, on note en revanche un tassement de la gauche, qui fait même moins bien, en Languedoc-Roussillon, qu'à l'échelle nationale (tableau 1). Pourtant, c'est dans ce contexte paradoxalement dépressif que la gauche obtient l'une de ses meilleures représentations à l'Assemblée, avec 20 députés sur 23. Parmi les causes d'un tel paradoxe, la présence du Front National s'impose avec force. Non seulement, avec Gilbert Collard, il conquiert l'un de ses deux sièges de député, mais sa présence dans 7 triangulaires (une dans l'Aude, trois dans le Gard, deux dans l'Hérault et une dans les Pyrénées-Orientales) s'avère fatale à la droite.

\footnotetext{
1 Nous assumons ici que le vote Le Pen, lors de la présidentielle de 2012, correspond au vote FN, tout en ayant clairement à l'esprit que la personnalisation du vote implique un dépassement de la seule dimension partisane de cette élection.
} 
Tableau 1. Les élections présidentielles 2012 en Languedoc-Roussillon

\begin{tabular}{|l|r|r|r|r|r|r|}
\hline Premier tour LR & \multicolumn{1}{|c|}{ Second tour LR } \\
\hline Candidat(e) & 7027 & 0,45 & 0,56 & & & \\
\hline Nathalie Arthaud & 17072 & 1,10 & 1,15 & & & \\
\hline Philippe Poutou & 35465 & 2,29 & 2,31 & & & \\
\hline Eva Joly & 204170 & 13,16 & 11,10 & & & \\
\hline Jean-Luc Mélenchon & 408664 & 26,34 & 28,63 & 756925 & 51,16 & 51,64 \\
\hline François Hollande & 672398 & 43,34 & 43,75 & & & \\
\hline Sous-Total Gauche & & & & & & \\
\hline & 363880 & 23,45 & 17,90 & & & \\
\hline Marine Le Pen & 384097 & 24,76 & 27,18 & 722690 & 48,84 & 48,36 \\
\hline Nicolas Sarkozy & 3482 & 0,22 & 0,25 & & & \\
\hline Jacques Cheminade & 105433 & 6,80 & 9,13 & & & \\
\hline François Bayrou & 22228 & 1,43 & 1,79 & & & \\
\hline Nicolas Dupont-Aignan & 1551518 & 100,00 & 100,0 & & & \\
\hline Total exprimés & 333619 & $17,4 \%$ & $20,5 \%$ & 330584 & & $19,65 \%$ \\
\hline Abstention & 28038 & $1,47 \%$ & $1,52 \%$ & 102793 & 701190 & $4,68 \%$ \\
& & & & $(5,4 \%)$ & & \\
\hline Blancs et nuls & 28,0 ) & & & & & \\
\hline
\end{tabular}

Source : élaboration personnelle d'après ministère de l'Intérieur

Tableau 2. Les députés du Languedoc-Roussillon en 2012

\begin{tabular}{|c|c|c|c|c|c|c|}
\hline Départ. & Circ. & Nom, Prénom & Parti & $\%$ & âge & Activité professionnelle \\
\hline Aude & 1 & Pérez Jean-Claude & PS & 61,0 & 48 & Permanent politique \\
\hline Aude & 2 & Fabre Marie-Hélène & PS & 56,8 & 61 & Permanent politique \\
\hline Aude & 3 & Dupré Jean-Paul & PS & 63,0 & 68 & Retraité privé (banque) \\
\hline Gard & 1 & Dumas Françoise & PS & 41,9 & 52 & Assistante sociale \\
\hline Gard & 2 & Collard Gilbert & FN & 42,8 & 64 & Avocat \\
\hline Gard & 3 & Prat Patrice & PS & 41,4 & 47 & Permanent politique \\
\hline Gard & 4 & Verdier Fabrice & PS & 52,1 & 42 & Permanent politique \\
\hline Gard & 5 & Dumas William & PS & 61,4 & 70 & Retraité privé (banque) \\
\hline Gard & 6 & Cavard Christophe & VEC & 43,1 & 42 & Éducateur spéc. public \\
\hline Hérault & 1 & Roumegas Jean-Louis & VEC & 50,1 & 50 & Enseignant Primaire \\
\hline Hérault & 2 & Le Dain Anne-Yvonne & PS & 66,4 & 57 & Cadre public (recherche) \\
\hline Hérault & 3 & Dombre Coste Fanny & PS & 54,8 & 56 & Artisan \\
\hline Hérault & 4 & Roig Frédéric & PS & 55,4 & 43 & Permanent politique \\
\hline Hérault & 5 & Mesquida Kléber & PS & 61,4 & 67 & Retraité public \\
\hline Hérault & 6 & Roqué Dolores & PS & 39,8 & 60 & Enseignant Secondaire \\
\hline Hérault & 7 & Denaja Sébastien & PS & 42,9 & 32 & Enseignant Universitaire \\
\hline Hérault & 8 & Assaf Christian & PS & 55,2 & 40 & Cadre Public \\
\hline Hérault & 9 & Vignal Patrick & PS & 50,7 & 54 & Chef d'entreprise \\
\hline Lozère & 1 & Morel-A-L'Huissier Pierre & UMP & 50,5 & 53 & Avocat \\
\hline Pyrénées-Or. & 1 & Cresta Jacques & DVG & 43,0 & 57 & Cadre privé (CAF) \\
\hline Pyrénées-Or. & 2 & Siré Fernand & UMP & 50,5 & 67 & Médecin \\
\hline Pyrénées-Or. & 3 & Neuville Ségolène & PS & 53,0 & 42 & Cadre public \\
\hline Pyrénées-Or. & 4 & Aylagas Pierre & PS & 55,4 & 70 & Retraité Enseignement \\
\hline
\end{tabular}

Source : élaboration personnelle d'après ministère de l'Intérieur

On voit que la nouvelle représentation languedocienne montre un certain renouvellement sociologique, avec une moyenne d'âge de 49,5 ans pour les nouveaux députés, contre 56 ans pour les sortants ${ }^{2} ; 6$ femmes contre une seule en 2007). On note aussi des changements dans les activités professionnelles, où le nombre de permanents

\footnotetext{
2 En 2007, la moyenne d'âge des 21 députés élus s'établissait à 55,6 ans.
} 
(5/23), directement issus de l'organisation partisane, est assez important. Ainsi, si l'on isole les huit élus de moins de 50 ans, outre les 5 permanents politiques, 3 travaillent dans la sphère publique comme universitaire, enseignant ou éducateur. Au renouvellement générationnel et de genre, on peut donc opposer un certain rétrécissement du recrutement socioprofessionnel. Pour 17 nouveaux députés, on n'en trouve que 4 (Gilbert Collard inclus) qui ne soient pas, de facto, déjà en position de cumul des mandats exécutifs locaux.

Enfin, sur un plan plus électoral, les succès obtenus par les candidat(e)s de gauche sont, dans 6 cas, très largement inférieurs à $50 \%$ des voix. Les triangulaires, qui en sont la cause, sont l'un des effets de l'enracinement du vote FN en Languedoc-Roussillon ${ }^{3}$, sur lequel nous allons axer notre papier.

À l'occasion de ces élections présidentielles, les débats font rage sur les espaces, les ressorts et les motivations du vote $\mathrm{FN}$, ainsi que sur son hypothétique changement de style (Dezé 2012). L'approche géographique en fait le symptôme d'une distance problématique à la ville (Bussi, Colange et Rivière 2011), une nouvelle périphérie, reléguée aux marges du modèle métropolitain (Guilluy 2010). Pour d'autres, le vote FN serait l'expression de crispations identitaires, que l'on retrouverait au sein d'espaces différents : périurbain, ruralité en déshérence, quartiers résidentiels et populaires des villes elles-mêmes. L'explication du vote FN suscite le retour de très anciennes querelles sociologiques, comme celles qui opposent les tenants d'approches culturalistes, sensibles aux traditions locales ou aux identités religieuses, par exemple, et les partisans de variables a priori plus quantifiables, telles que la catégorie sociale, les niveaux scolaires ou de revenus, etc.

Pour d'autres encore, à commencer par le Front National lui-même, le vote Le Pen signifierait que «le peuple s'invite à la table des élites », autrement dit que les «petits, les sans-grades » constitueraient aujourd'hui le « vrai » vote populaire.

L'enquête auprès des électeurs du Front National entraine un certain scepticisme quant à ces théories, lorsqu'elles se prétendent seules à pouvoir expliquer un tel comportement politique (Girard 2012). Si le vote pour les candidats d'extrême-droite reste mal connu, fluctuant d'une élection à l'autre (Mayer 2002, Lehingue 2003 ; 2011), c'est aussi qu'il obéit à des causes en partie distinctes d'un territoire politique à l'autre. Comme toute approche localisée des comportements électoraux, nous faisons l'hypothèse que le lieu «dit» l'opinion, et qu'il existe un lien entre tel type de comportement et telle configuration locale, même si nous étudions des votes agrégés. Ainsi, lorsque nous constatons que le vote FN est plus élevé dans les villes où les catégories moyennes sont sur-représentées, cela ne signifie pas que chaque ménage de classe moyenne vote FN. En termes purement statistiques, l'observation, même fine (au niveau des bureaux), des votes ne suffit pas à construire un lien de causalité (Braconnier 2011). Mais cette observation, accompagnée de modalités plus qualitatives d'enquêtes (entretiens auprès des électeurs), de données régionales sur une certaine durée (20 ans), aboutit à un niveau de cohérence suffisamment pertinent pour valider certaines hypothèses et surtout en invalider d'autres. Les biais qu'elle comporte sont sans doute, dans ce cas, moins importants que n'importe quelle étude par sondage. Dans ce papier, nous allons donc discuter deux thèses. La première est celle qui fait du vote FN la traduction inéluctable d'un malaise néo-périphérique. La seconde est celle qui fait de ce

\footnotetext{
37 triangulaires, cela représente le quart du nombre total de triangulaires avec la présence d'un candidat du FN en 2012 (28). Le Languedoc-Roussillon pèse 4\% du nombre total de députés.
} 
même vote l'expression désormais dominante des dominés. La première partie s'intéresse à caractériser les zones de force du vote FN et ce qu'elles nous disent de ses dynamiques régionales. La seconde partie concerne les caractéristiques du vote dans les zones les plus populaires de la région ${ }^{4}$.

\section{La diffusion du vote FN en Languedoc-Roussillon}

Où les zones de force du FN dans la région Languedoc-Roussillon se situent-elles en 2012 ? Nous allons ici constater qu'elles ne diffèrent pas énormément de celles déjà identifiées depuis les années 1990 (Négrier 2001, Alliès, Baraize \& Négrier 2002, Négrier \& Jourda 2010, Négrier, Jourda \& Ratinaud 2011). La ruralisation du vote FN, qui n'est pas totalement nouvelle, mais prend une ampleur cette fois remarquée, n'infirme pas une implantation privilégiée de ce dernier dans ce que l'on peut désormais appeler ses bastions. Ensuite, nous verrons qu'elle entretient une relation paradoxale à l'égard de la couleur politique des municipalités. Enfin, nous discuterons la thèse d'un vote comme protestation spatialisée, en avançant au contraire celle de l'expression d'une certaine conviction.

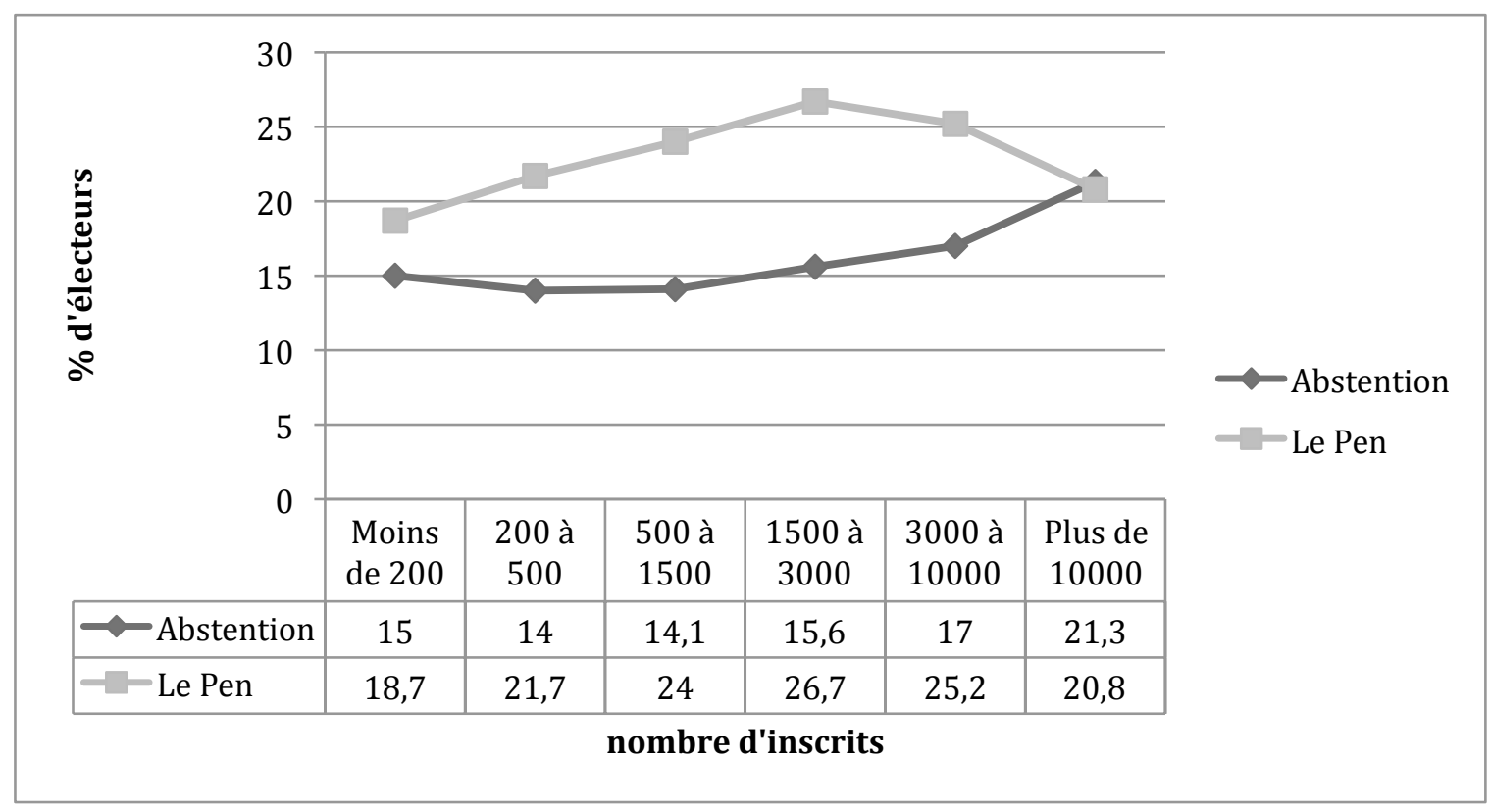

Graphique 1. Le vote FN en Languedoc-Roussillon par taille des communes ${ }^{5}$

\section{A. Le vote FN : ses bastions, sa diffusion rurale}

Ce graphique nous montre une figure en apparence assez classique du vote FN, que l'on a constatée lors de tous les scrutins en Languedoc-Roussillon: les zones de force du

\footnotetext{
${ }^{4}$ Nous tenons à remercier les responsables des élections dans les préfectures du Languedoc-Roussillon, ainsi que ceux des villes avec lesquels nous avons pu échanger sur la géographie électorale précise des ZUS

5 Le choix des seuils se justifie par des finalités de comparaison avec des données analysées de la même manière à l'occasion des élections régionales de 2010 (Négrier, Jourda \& Ratinaud 2011).
} 
Front National se situent dans les communes comptant moins de 10000 inscrits, mais plus de 1500. Les petites communes, qui incarnent (sauf exception) le monde rural (moins de 200 inscrits) situent en moyenne le FN à moins de 17,9\%. Les communes de plus de 10000 inscrits la situent à 20,8\%. Ces scores sont considérables. Pour donner un seul exemple, le niveau atteint par Marine Le Pen en Lozère $(17,3 \%)$ est supérieur au score prêté à la candidate frontiste par les sondages pré-électoraux. C'est, pour la petite histoire, à Fournels, le fief du député Pierre Morel à l'Huissier, que le FN fait son meilleur score lozérien : 28,6\%. Si on les compare, mutatis mutandis, aux scores du FN lors des élections régionales de 2010 , le FN passe respectivement de $8,2 \%$ des voix à $18,7 \%$ dans les communes de moins de 200 habitants; et de 10,4\% à 21,7\% dans les communes comptant entre 200 et 500 inscrits. Cela représente une progression en pourcentages de plus de $100 \%$, qui plus est (cf. graphique) dans les zones où la participation est la plus importante à ce scrutin, comme à celui des élections régionales d'ailleurs. Dans les communes comprises entre 1500 et 10000 inscrits, le FN dépasse le quart des suffrages exprimés. Ces communes pèsent à elles seules près de la moitié des électeurs languedociens. Le FN y pesait $14 \%$ des voix en 2010. C'est dans les moyennes et grandes villes de la région (+ de 10000 inscrits), où il passe tout de même de 12,4\% à 20,8\%, qu'il progresse le moins. On notera, à l'instar de plusieurs autres régions, que le Languedoc-Roussillon étend la diffusion du vote FN au-delà des bastions classiques (petite Camargue et agglomération biterroise, littoral, périurbain en général).

\section{B. Vote FN et territoires politiques}

Le FN obtient plus de 30\% des suffrages dans 160 communes sur les 1545 que compte la région. Si l'on observe de plus près les communes de plus de 3500 habitants, où les élections municipales se déroulaient en 2008 au scrutin de listes bloquées, propices à une identification droite/gauche plus aisée que dans les petites communes, on peut faire les constats suivants :

1. Les scores du FN sont relativement indifférents à la couleur politique des conseils municipaux. Ici, nous avons comptabilisé à droite les votes pour Nicolas Sarkozy, Marine Le Pen, Nicolas Dupont-Aignan et François Bayrou. Entre les 24,8\% en moyenne pour le FN dans les communes dirigées par des conseils de gauche et les $25,1 \%$ dans celles de droite, l'écart est négligeable.

Tableau 3. Le vote des communes de plus de 3500 habitants en Languedoc-Roussillon

\begin{tabular}{|c|c|c|c|c|}
\hline Communes & inscrits & votants & FN & droite \\
\hline Droite (64 communes) & 592420 & 82,9 & 25,1 & 60,0 \\
\hline Gauche (67 communes) & 561546 & 82,4 & 24,8 & 56,6 \\
\hline
\end{tabular}

2. On constate que la droite est bien placée dans les 64 communes qu'elle gouverne, mais aussi qu'elle dépasse le plus souvent la barre des 56,8\%, c'est à dire qu'elle est majoritaire sans même l'appui des $6,8 \%$ des voix obtenus en LanguedocRoussillon par François Bayrou. Au total, la gauche ne dépasse $50 \%$ des voix que dans 12 communes sur 131 de plus de 3500 habitants.

3. La singularité du contexte, pour cette droite, est de devoir compter sur une extrême-droite très présente. Dans 38 communes, le FN atteint presque ou dépasse les $50 \%$ du total droite (Bayrou et Dupont-Aignan inclus). 
4. Les 28 communes de plus de 3500 habitants où le FN dépasse $30 \%$ des votants (ou les frôle à moins de 1\%) ne sont pas que les communes gardoises (13) dont on a tant parlé ces derniers jours: Saint-Gilles, Beaucaire, Vauvert, etc. On y compte aussi deux audoises (dont Port-la-Nouvelle, record de sa catégorie avec 37,2\%), 8 héraultaises (dont Vias, Villeneuve-lez-Béziers, Montady) et 4 roussillonnaises (dont Le Barcarès ou Pia). Elles représentent 136370 électeurs inscrits (soit 7,1\% de l'électorat languedocien).

5. Ces communes ne sont pas particulièrement éloignées des grands centres urbains (Milhaud, Villeneuve-lez-Béziers), ni toujours voisines (Saint-Gilles, Portla-Nouvelle, Montady). Elles ne sont ni particulièrement riches, ni toutes très pauvres.

6. Les communes les plus riches en revenus des foyers fiscaux votent assez peu pour le Front National : Saint-Clément de Rivière, Saint-Gély du Fesc, Castelnaule-lez ou Clapiers, dans l'Hérault, Les Angles ou Vileneuve-lez-Avignon dans le Gard, par exemple.

7. Les communes les plus pauvres ne «tombent» pas systématiquement dans l'escarcelle du FN pour autant: la Grand Combe (20\%) ou Bédarieux (19,2\%) accordent moins de voix au FN que leur moyenne départementale, alors que leur identité populaire et socialement dégradée est avérée par les indicateurs de revenu, de chômage, de précarité sociale.

En conséquence, la géographie du vote FN tend à rendre peu pertinente l'idée qu'il y aurait une " mauvaise distance » à la ville pour expliquer l'intensité de ce vote, ou qu'il serait l'expression inéluctable d'une souffrance particulière, repérable par exemple au niveau de revenu. Sur ce dernier point cependant, les espaces de forte intensité frontiste coïncident le plus souvent avec des communes moyennes, périurbaines, qui ont connu une forte expansion démographique, où les ménages aux revenus moyens se sont installés, sans toujours pouvoir assumer, en pratique, le train de vie qui correspondait à l'aspiration à devenir propriétaire en zone résidentielle. Dans ces communes en effet, la part des propriétaires de leur logement (par opposition aux locataires) est presque systématiquement supérieur à la moyenne, même si le prix municipal du foncier n'est pas toujours parmi les plus élevés de la région, au contraire. Certes, quelques communes aux indicateurs de revenu et de statut social favorables accordent cependant leurs suffrages en nombre au FN. Ainsi les habitants de Bouillargues $(27,8 \%$ FN) disposent d'un revenu moyen supérieur de $25 \%$ et connaissent un chômage inférieur de $40 \%$ à la moyenne du Gard, et sont à 75\% propriétaires de leur logement.

Mais plus globalement, ce vote dessine l'image d'une classe moyenne au patrimoine récent, fragile, dont on craint en contexte de crise la valeur durable et la transmissibilité. La crispation identitaire est, sans doute d'abord, dans cette incertitude. Elle répond, comme en écho, à celle qui frappe les désormais rares natifs de ces lieux, qui peuvent y subir une dépossession symbolique (paysagère, culturelle), alors même qu'ils sont, mieux que les nouveaux arrivants, représentés au sein des conseils municipaux. Cette incertitude n'est pas mécaniquement fonction de la distance au centre métropolitain, dans une région où la pression démographique et foncière s'étend par ondes spéculatives plus ou moins intenses selon les terroirs. Elle n'est ni mécanique, ni indifférente aux contextes culturels, historiques, économiques qui marquent les territoires. La «dépossession» symbolique n'est pas moins vive dans les quartiers 
populaires d'anciennes cités minières du Gard et pourtant, on le verra ensuite, le vote FN y est (encore ?) limité.

\section{Vote de protestation, de conviction?}

En faisant du vote FN l'expression géographique et sociale d'une frustration urbaine, on perd de vue un double problème qui en assure l'empreinte territoriale : une conviction diffuse ; l'absence de réponse construite à cette conviction diffuse.

Dans les registres de justification du vote FN, tels que nous avons pu les observer auprès d'électeurs languedociens ${ }^{6}$, entrent bien sûr la frustration dont nous parlons depuis les années 1990, et ce désarroi pavillonnaire d'une région en intense transformation démographique. Il s'agit là d'éléments composites, qui vont du sentiment de dépossession de l'exploitant agricole retraité, dont la maigre pension (autour de 700 euros mensuels) cadre mal avec la représentation de soi; à la désillusion du jeune ménage, installé très loin des lieux de travail, d'apprentissage et de loisirs, subissant avec colère le renchérissement et le délitement d'un projet de vie.

Mais ces individus ne sont pas inertes face à ces dépossessions, à ces désillusions. Ils sont d'abord construits par des cultures familiales, qui autorisent un chemin vers le vote FN, ou en font au contraire un tabou. Ils font ensuite preuve de conviction dans le choix du vote extrême, d'autant plus qu'elle se fonde sur la déclaration de n'être convaincu par aucune des offres politiques « classiques ». Certains - les plus diplômés ou installés - font ainsi référence à l'autorité qui aurait disparu, à l'identité de la France qui serait menacée, à la pression fiscale excessive, au capitalisme apatride qu'il faudrait bouter hors des frontières. D'autres voient la perte et la menace à une échelle plus limitée, qui peut être celle du profiteur, de l'assisté, de l'immigré, de l'étranger plus généralement, qui envahirait l'espace avec ses bruits, ses odeurs, ses prétentions à l'emploi et à l'aide sociale. Ici se trouve une expression plus populaire d'un même rapport pathologique à l'altérité. Celle-ci est construite comme un problème et vécue comme une menace. C'est cette problématisation, plus ou moins élaborée, d'un bouc émissaire (l'arabe, l'anglais, mais aussi le nîmois, le montpelliérain, ... le voisin), qui fait «conviction ». Elle ne rencontre pas nécessairement, dans les réseaux de sociabilité locale, dans les politiques municipales, des réponses politiques à ces « arguments » diffus. Et l'analyse qui consiste à « ne pas accepter mais comprendre » un vote qui traduirait inéluctablement un état socio-économique ou spatial, fait partie de ce halo de respect où prospère le "panache » frontiste.

C'est sur ce point que le positionnement de Jean-Luc Mélenchon s'est révélé intéressant, en ce qu'il a précisément constitué une réponse relativement simple, déterminée, et susceptible d'être relayée dans les échanges politiques, à la fois informels et passionnés, qui nourrissent ces réseaux: associations de chasse, cafés, cercles d'amis divers. Le "panache » Mélenchon s'est opposé à celui de Marine Le Pen, au point - selon nos observations de terrain - de faire basculer des tentations FN vers la gauche, au premier tour, plus sûrement que le changement raisonnable qu'incarnait François Hollande. L'épreuve rhétorique, en partie réussie, n'est sans doute qu'une étape dans cette

\footnotetext{
6 Nous réalisons depuis 2007 une enquête au long cours dans un village languedocien, auprès des électrices et électeurs, afin d'approfondir la connaissance que nous avons du rapport ordinaire à la politique, à l'offre et aux comportements électoraux, aux registres de justification qui le fondent. La publication des premiers résultats se fera en 2013.
} 
réponse politique à la diffusion de la conviction frontiste. L'autre étape, cruciale, passe par l'action publique et la confrontation politique au quotidien. Ici, l'issue est plus incertaine et dépend des configurations locales. La meilleure preuve en est sans doute apportée par les reports de voix entre premier et second tour des élections présidentielles.

Comment en effet ce vote, où il faut voir de la conviction et non simplement une protestation générique, s'oriente-t-il au second tour? On peut schématiquement observer que le report sur le président sortant est très favorable dans les communes gérées par la droite. Il en est ainsi de Palavas : près de 65\% pour Nicolas Sarkozy au second tour, à peine moins que le total de toutes les voix de droite, Bayrou inclus, au premier tour. Mais c'est aussi le cas de Fabrègues ou Saint-Clément de Rivière, dans l'agglomération montpelliéraine, ou de Port-Vendres et Canet-en-Roussillon sur la côte roussillonnaise. Cette règle souffre quelques exceptions, notamment là où la gauche municipale est inscrite dans un bastion FN: Villeneuve-lez-Béziers et Thézan-lezBéziers, pour le biterrois, Argelès-sur-Mer dans les Pyrénées-Orientales, sont des villes de gauche où la droite l'emporte nettement au second tour. Ce report est, cependant, moins automatique dans les communes qui ont une culture politique plus orientée à gauche: Aramon pour la petite Camargue gardoise, Villeneuve-lès-Maguelone ou Frontignan pour l'Hérault, Elne pour les Pyrénées Orientales sont des communes de gauche où les droites, majoritaires en cumul au premier tour, se rallient mal à Nicolas Sarkozy au second tour et où ce dernier est devancé par François Hollande. Cette situation peut être élargie aux communes de plus petite taille, situées en zone rurale, où le FN a fait une percée remarquée. Un seul exemple, en territoire cévenol, jadis considéré comme rétif à l'extrême-droite: Saint-Cécile d'Andorge, près de la Grand Combe. Le FN y réalise près de $35 \%$ des voix au premier tour. Les droites y totalisent $58 \%$ des voix. Au second tour, Nicolas Sarkozy dépasse à peine $40 \%$ des suffrages. Les $10 \%$ de votes blancs et nuls ne suffisent pas à expliquer cet écart, ni l'abstention, de moins de $2 \%$ inférieure au second tour.

Résumons ce premier point :

a) le FN retrouve en 2012 des territoires d'influence où il était déjà puissant dans les années 1990, tout en étendant son influence en zone rurale. Ses bastions restent des communes entre 1500 et 10000 habitants, en récente et forte croissance démographique, proches des centres métropolitains, où l'on est en général propriétaire de son logement et où le niveau de revenu est moyen. Les communes riches votent peu Le Pen, tout comme les pauvres.

b) cette diffusion est relativement indifférente, au premier tour, à la couleur politique des municipalités, notamment dans les communes de plus de 3500 habitants. Cela ne signifie nullement que le vote FN ne soit que l'expression d'une protestation diffuse ou d'un malaise périurbain. C'est aussi un vote de conviction, même si celle-ci est réversible.

c) Au second tour, beaucoup de communes de gauche retrouvent une majorité en faveur de François Hollande, en raison d'une défection assez élevée, parfois, de l'électorat Le Pen à l'égard de Nicolas Sarkozy. Cette défiance est prononcée dans les zones rurales ou industrielles de culture de gauche. Elle est bien moins évidente dans les bastions FN (biterrois, petite Camargue, plaine roussillonnaise, littoral plus généralement), où droite et extrême-droite s'additionnent plus 
volontiers au second tour. Jusqu'à un certain point, on peut donc identifier deux votes FN.

d) La différenciation territoriale de l'implantation du vote Le Pen, même en utilisant différentes lunettes (couleur politique, données urbaines, niveaux de revenus), rend plausible une variable liée à la culture politique territoriale. Il s'agit moins de se référer à une tradition inerte qu'à sa sédimentation de pratiques qui correspondent à une culture territorialisée : encadrement militant et croyance dans les Cévennes minières et protestantes, violence latente des éléments et des représentations de l'altérité dans la petite Camargue, intériorisation du déclassement sociétal dans l'univers biterrois, etc. Ces variables, par nature plus délicates à manipuler, ne doivent donc être convoquées que lorsque les autres ont épuisé leur efficacité, ce qui reste fréquent. C'est tout l'intérêt d'une géopolitique du vote FN que d'en restituer la complexité.

Jusqu'à présent, nous avons plus souvent mentionné le vote d'univers périurbains, littoraux, voire ruraux que celui des villes. Or c'est dans celles-ci que se concentre une grande partie des difficultés sociales, économiques. Pour le dire brutalement, c'est dans les villes qu'on trouve le plus facilement les pauvres, et qu'il est donc possible d'examiner leur rapport au vote en général, et au FN en particulier. Eu égard au double discours du FN (le " peuple s'invite à la table des élites en votant Le Pen ») et de certains cercles de gauche («Les catégories populaires ne sont plus au cœur de l'électorat de gauche ${ }^{7}$ ). Il est évidemment intéressant de le scruter de plus près.

\section{Quel vote des pauvres, des sans grade?}

Le vote FN ne se concentre pas forcément là où la richesse est la plus manifeste. Mais est-il pour autant puissant là où les indicateurs de précarité, de difficulté sociale, de pauvreté sont les plus élevés?

Tableau 4 : le vote des bureaux en Zone Urbaine Sensible en Languedoc-Roussillon

\begin{tabular}{|c|c|c|c|}
\hline Données & Votants & Score FN & Total Droites* \\
\hline Montpellier Total & 77,6 & 13,7 & 44,1 \\
\hline Montpellier ZUS & 74,6 & 13,8 & 36,0 \\
\hline Béziers Total & 78,6 & 25,7 & 60,8 \\
\hline Béziers ZUS & 79,8 & 28,1 & 55,4 \\
\hline Sète Total & 75,9 & 22,5 & 57,6 \\
\hline Sète ZUS & 76,8 & 24,7 & 40,5 \\
\hline Lunel Total & 80,0 & 27,0 & 58,5 \\
\hline Lunel ZUS & 77,7 & 27,0 & 53,1 \\
\hline Alès Total & 78,3 & 20,9 & 52,2 \\
\hline Alès ZUS & 67,6 & 10,9 & 21,3 \\
\hline Nîmes Total & 78,6 & 20,6 & 55,8 \\
\hline Nîmes ZUS & 69,0 & 17,1 & 36,5 \\
\hline
\end{tabular}

\footnotetext{
7 On fait bien sûr ici référence au rapport polémique du laboratoire d'idées Terra Nova, qui indiquait dans un rapport publié en mai 2011 : «Il n'est pas possible aujourd'hui pour la gauche de chercher à restaurer sa coalition historique de classe : la classe ouvrière n'est plus le coeur du vote de gauche, elle n'est plus en phase avec l'ensemble de ses valeurs, elle ne peut plus être comme elle l'a été le moteur entraînant la constitution de la majorité électorale de la gauche. La volonté pour la gauche de mettre en oeuvre une stratégie de classe autour de la classe ouvrière, et plus globalement des classes populaires, nécessiterait de renoncer à ses valeurs culturelles, c'est-à-dire de rompre avec la social-démocratie. » (Terra Nova, 2011).
} 


\begin{tabular}{|c|c|c|c|}
\hline La Grand Combe Total & 74,5 & 20,4 & 41,2 \\
\hline La Grand Combe ZUS & 76,6 & 13,6 & 24,7 \\
\hline Saint Gilles total & 79,6 & 35,4 & 61,8 \\
\hline Saint-Gilles ZUS & 75,8 & 15,8 & 30,6 \\
\hline Beaucaire total & 79,4 & 34,1 & 61,8 \\
\hline Beaucaire ZUS & 77,2 & 33,6 & 58,9 \\
\hline Bagnols/Cèze Total & 75,5 & 25,4 & 57,3 \\
\hline Bagnols/Cèze ZUS & 68,9 & 22,4 & 47,2 \\
\hline Narbonne total & 78,3 & 22,1 & 54,7 \\
\hline Narbonne ZUS & 77,8 & 22,0 & 43,9 \\
\hline Carcassonne total & 79,5 & 22,5 & 52,8 \\
\hline Carcassonne ZUS & 78,9 & 23,2 & 47,4 \\
\hline Perpignan total & 75,9 & 22,5 & 57,6 \\
\hline Perpignan ZUS & 72,4 & 23,5 & 48,0 \\
\hline
\end{tabular}

* Le total Droites est l'addition des scores obtenus au premier tour par Nicolas Sarkozy, Marine Le Pen, François Bayrou et Nicolas Dupont-Aignan.

Le vote FN est-il celui du prolétaire languedocien du XXIème siècle ? Pour le montrer, nous nous sommes intéressés au vote dans les Zones Urbaines Sensibles (ZUS) qui ont la particularité de concentrer ces «petits et sans grade » et de les identifier à partir d'une batterie de critères objectifs, ceux qui font précisément que les quartiers concernés seront concernés par la politique de la ville. Nous avons pour cela comparé le vote des villes avec celui des 91 bureaux de vote, dans ces villes, situés en périmètre ZUS (tableau 4). Le résultat est particulièrement intéressant.

Cette observation des 13 villes comportant un quartier ZUS nous permet de faire les cinq constats suivants : a) on y participe un peu moins que la moyenne, sauf dans trois cas ; b) on y vote moins FN, sauf dans trois cas ; c) on y vote moins à droite, dans tous les cas ; d) ces quartiers ont un comportement qui diffère selon les configurations locales ; e) les pratiques politiques ont un impact sur les orientations de vote. Ces constats, fondés sur l'analyse du premier tour des présidentielles, sont cohérents avec celle du second tour.

Le premier enseignement concerne le taux de participation : il est inférieur à la moyenne de la ville, et parfois dans des proportions très importantes, comme à Bagnols-sur-Cèze $(-7 \%)$ ou Alès $(-11 \%)$. Le peuple s'est donc moins invité que les autres... au bureau de vote. Il est cependant important de constater, pour cette élection présidentielle, que le différentiel de participation entre les bureaux populaires et les autres est beaucoup moins élevé que pour d'autres élections. Ainsi, lors des élections cantonales de 2011, il a pu dépasser les $20 \%$ de participation en moins pour les bureaux populaires de Nîmes ou de Montpellier. Il faut enfin constater que cette règle de moindre participation connaît des exceptions : Béziers, Sète, La Grand Combe. Dans les deux premiers cas, cela est d'abord dû à une certaine mixité sociale d'une partie des bureaux de vote. Ainsi à Béziers, si l'on extrait des dix bureaux de vote situés en ZUS les 4 qui concernent le plus un habitat pavillonnaire (où l'on vote plus, et plus à droite et à l'extrême-droite), le niveau de participation des 6 restants devient inférieur à la moyenne. Dans le cas de la Grand Combe, le bureau de vote situé en ZUS renvoie à un quartier très défavorisé mais dont la population change relativement peu, et dont la taille est modeste (471 inscrits). Il est donc plus facile d'y déployer des formes de mobilisation électorale fondées sur l'interconnaissance, les relais publics locaux, dans une ville gérée par la gauche depuis très longtemps. 
Le deuxième constat concerne l'intensité du vote FN. Celui-ci est globalement moins présent dans ces zones que dans le reste de la ville. L'écart est parfois mince. À Béziers, seule ville dans ce cas, il est même légèrement supérieur. Nous y reviendrons. À Sète, à Lunel ou à Montpellier, tout comme à Perpignan, Carcassonne ou Narbonne, il est aussi haut, ou bas que dans le reste de la ville. À Alès, La Grand Combe ou Saint-Gilles, il accuse un retard beaucoup plus important $(-10 \%$ à Alès). Pour prendre un chiffre emblématique, la ZUS de Saint-Gilles n'accorde « que » $15,8 \%$, dans une ville qui atteint en moyenne (ZUS comprise, donc) le chiffre record de $35,4 \%$ des voix.

Troisième enseignement: dans sa diversité, la droite est distancée dans ces zones sensibles. Si le FN pâtit du vote populaire le plus caractéristique, au lieu d'en être l'expression privilégiée, la droite en est encore plus nettement victime. Les quartiers en ZUS accordent nettement moins de suffrages aux droites, prises globalement : autour de $5 \%$ de moins à Béziers, Carcassonne ou Lunel ; entre $8 \%$ et $10 \%$ de moins à Montpellier, Bagnols sur Cèze, Perpignan, et jusqu'à $-10 \%$ voire plus à Nîmes, Alès ou la Grand Combe.

Cette analyse doit cependant compter avec la diversité des quartiers en ZUS au sein des villes, et à la façon dont les bureaux de vote en rendent compte. Deux exemples : les ZUS montpelliéraines ont des taux assez variables, notamment en fonction de la part de logement collectif et de celle, parfois inexistante mais parfois plus importante, des résidences et lotissements individuels. En règle générale, plus les bureaux de vote renvoient à du logement social et collectif, plus le vote FN régresse. Si la proportion de logements individuels, de type pavillonnaire, augmente (même en périmètre ZUS), le vote à droite et, singulièrement FN, croît aussi. C'est un constat que l'on peut faire sur 4 des 10 bureaux de votes situés au cœur du quartier de la Devèze. Les zones pavillonnaires qui entourent la Devèze, tout comme celles qui sont comprises à proximité des zones urbaines sensibles des autres villes ont des scores FN largement supérieurs à la moyenne ${ }^{8}$. Ce résultat nous renvoie aux premières analyses du vote FN en termes d' " effet de halo ", notamment lorsque Pascal Perrineau (1988) contestait, avec d'autres (Mayer 1987, Martin 1996), la coïncidence entre le vote FN et la présence d'étrangers à l'échelle départementale (Le Bras 1986 / 1996). C'est moins le fait de vivre «dans » la pauvreté qui peut provoquer le vote d'extrême-droite que celui d'en être immédiatement voisin.

Enfin, une comparaison interurbaine entre les quartiers en ZUS à Béziers, et ceux de Montpellier, incite à penser que ces variables sont insuffisantes pour comprendre les dynamiques de vote en quartier populaire. Dans chacun des deux, la participation se situe à plus de $75 \%$ des voix, ce qui est tout de même respectable pour qui se souvient, dans ces bureaux de vote, des maigres taux de participation lors des 5 scrutins qui ont eu lieu entre les deux élections présidentielles (législatives, municipales, cantonales européennes et régionales). À Montpellier, ce taux de participation entraine un score très élevé de la gauche, et corrélativement faible de la droite, qui culmine (Bayrou

\footnotetext{
8 Ces constats tiennent notamment à l'évolution urbaine, et peuvent introduire des biais dans l'assimilation entre l'implantation du bureau de vote en périmètre ZUS, d'une part, et la réalité urbaine à laquelle se réfère la majorité de la population qui y vote. Par exemple à Beaucaire, les bureaux 7 et 8 sont implantés en ZUS, dans ce qui représentait, dans les années 1980-90, les confins de la ville, constitués d'habitat HLM. Depuis, l'urbanisation s'est étendue, en habitat pavillonnaire, à proximité d'un hypermarché. Le bureau de vote le plus représentatif de ce nouveau quartier, bien que toujours localisé en ZUS, regroupe une forte majorité d'habitants de cette nouvelle urbanisation. Il vote plus, plus à droite, et plus FN.
} 
inclus) à $36 \%$ dans ces quartiers, contre $44 \%$ sur la ville entière. À Béziers, les bureaux de la Devèze atteignent $55,4 \%$ de vote à droite contre $60,8 \%$ en moyenne sur la ville. Le quartier populaire, tout en étant plus orienté vers le FN que la moyenne $(27,4 \%$ contre $25,7 \%$ en moyenne à Béziers), reste assez fortement marqué à droite. Pour comprendre cet écart, on peut faire référence à deux phénomènes.

a. La présence de l'appareil politique et institutionnel dans chacun des quartiers: les réseaux d'acteurs et relais municipaux dans le cas de Béziers, qui fonctionnent à droite; leurs homologues, ainsi que les dispositifs départementaux fonctionnent à gauche dans le cas des ZUS montpelliéraines, et singulièrement le quartier de la Mosson, le fief du président du conseil général de l'Hérault.

b. Ces réseaux et relais ont été mobilisés en 2011 à l'occasion d'élections cantonales particulièrement disputées: le conseiller général sortant socialiste, Jean-Michel Duplaa, a eu fort à faire pour l'emporter au second tour, et de très peu, face à son challenger du FN, Guillaume Vouzellaud. On peut penser que ces relais ont pu être plus facilement réactivés qu'ailleurs, ce qui expliquerait à la fois le bon niveau de la participation et l'orientation plus à gauche de ces bureaux biterrois par rapport à la moyenne de la ville.

L'observation du second tour est un utile prolongement de ces leçons. Sur 91 bureaux de vote, Nicolas Sarkozy n'est en tête que dans 7 d'entre eux, presque toujours d'extrême justesse, sauf à Beaucaire, dans deux bureaux. Ces derniers sont tous situés en villes de droite (Perpignan, Béziers, Nîmes et Beaucaire). En globalité, François Hollande atteint $64 \%$ des voix, contre $36 \%$ à Nicolas Sarkozy, alors que le total des droites, au premier tour, était de près de $44 \%$. Cela signifie que le vote Le Pen en zone populaire est plus réticent à l'égard de la droite " gouvernementale » que ne le sont les zones périurbaines. Ce n'est pas que les votes blancs et nuls y soient supérieurs à la moyenne, au contraire $(5,2 \%)$. L'abstention y est en revanche un autre signe de défiance ou de décrochage à l'égard du vote central du système politique français $(24,5 \%$, soit $50 \%$ d'abstention audessus de la moyenne régionale).

En résumé, pour comprendre la géographie du vote $\mathrm{FN}$, il faut manipuler une combinaison de facteurs et non une variable unique, qu'elle soit culturelle, sociale ou topographique. La structure urbaine, les niveaux de revenu ont leur part dans le phénomène. Le vote FN n'est peut-être pas le vote des riches. Mais il n'est certes pas celui des pauvres, pourrait-on dire très schématiquement. La pratique politique, la présence de relais territorialisés comptent aussi. Nous l'avons vu pour les quartiers, mais nous l'avons déjà souvent mis en évidence depuis 1986: l'intégration du FN comme interlocuteur puis allié dans des stratégies majoritaires au Conseil Régional, en 1986 puis en 1998, ne sont pas sans influence sur la légitimation du FN dans l'offre politique languedocienne. Son retour à un haut niveau dans le biterrois ainsi que dans la petite Camargue - c'est à dire là où le FN sous direction mégrétiste avait le plus joué la carte de l'enracinement local, dans les années 1990 - montrent que les pratiques politiques laissent des traces, légitiment des trajectoires, consolident des bastions ${ }^{9}$.

\footnotetext{
${ }^{9}$ Nous n'évoquons pas ici les variables plus physiques, culturelles et historiques qui sont, notamment dans la plaine gardoise, parfois mentionnées : la violence relative du climat, avec ses plaines venteuses, ses vidourlades, ses étés caniculaires ; la tradition camarguaise et son esprit cocardier, tourné vers un entre-soi hostile à l'allochtone; le rappel des massacres d'étrangers, et notamment à Aigues-Mortes en 1893 à l'égard des italiens qui (déjà) étaient accusés de
} 
Tout l'enjeu, autour des élections législatives, était là. Ceux qui pensent que le FN demeure structurellement handicapé dans le système de la Vème République font référence à quatre facteurs :

- le FN a un électorat extrêmement fluctuant, dont l'intermittence altère la capacité à s'enraciner localement.

- Le FN n'est réellement fort que lors des élections présidentielles, où sa composante populiste joue à plein autour de la personne du leader; il voit son capital électoral fondre lors d'élections suivantes, même proches dans le temps.

- Une élection présidentielle permet de dissimuler, derrière la parole du chef, les divisions qui continuent d'agiter le parti, et qui se révèlent, au contraire, à l'occasion des autres moments de la vie politique. Le FN peut être la proie de dissidences ou de défections dans les circonscriptions.

- Lors d'élections législatives, le FN ne dispose que d'une capacité de nuire, incapable qu'il est de s'inscrire dans des coalitions majoritaires de second tour, avec une droite qui en exclut la perspective.

De l'autre côté, certaines signent pourraient laisser croire à une évolution sur chacun des points :

- le FN s'appuie aujourd'hui sur des cadres relativement jeunes, formés à l'action politique, qui redonnent au parti l'encadrement qu'il avait perdu lors de la scission de 1998. Cet encadrement permet au FN d'envisager une stabilisation de son électorat

- le FN a désigné l'échéance législative comme un moment clef de sa nouvelle stratégie politique de conquête, jouant sur une exacerbation des clivages postélectoraux au sein de l'UMP. Il a donc « accroché » un électorat plus important qu'à l'accoutumée.

- La perspective d'atteindre ou de consolider une part majoritaire de la droite, en Languedoc-Roussillon, permet de réguler (au moins provisoirement) les tensions au sein d'un appareil orienté vers plusieurs trophées électoraux désormais à portée de main.

- Le souci de préservation à court et moyen termes de positions de pouvoir peut conduire la droite (ou une partie de ses élus, en francs tireurs ou se déliant de leur loyauté partisane dans un contexte de règlement de comptes) à nuancer ou réviser son rejet de tout accord avec le FN. La droite languedocienne dispose d'une expérience à ce sujet. Certains acteurs, aujourd'hui parlementaires et édiles, l'on personnellement vécue par deux fois, en 1986 et 1998, pour bâtir une majorité au Conseil Régional.

\section{Conclusion}

Plutôt que de chercher de façon systématique à coller aux électeurs des étiquettes indélébiles et à les situer dans des cases dont ils ne pourront s'extraire, notre double analyse s'est intéressée à la fois à l'enracinement territorial du vote et aux conditions de

priver d'emploi la main d'œuvre locale (Noiriel 2010). Ces variables sont délicates à manier, et encore plus à construire en "gène » territorial. Pour se limiter au Gard, alors que l'on considère que les Cévennes et leurs bassins miniers sont construits sur des mentalités toutes distinctes (solidarité, progressisme, protestantisme), il faut se souvenir que les mineurs se dressaient aussi, dès 1848, contre les étrangers (Huard 2008). Peser le poids de ces facteurs excède le cadre de ce texte, sans pour autant leur dénier toute importance. 
son émancipation. L'explication territoriale, sociale, mentale du vote FN correspond désormais à des constantes, voire à des bastions. Leur importance est à la mesure de la faiblesse de l'activité proprement militante qui les caractérise, pour un parti qui n'a pas encore opéré sa véritable mue notabiliaire, et qui ne la fera peut-être pas.

La confirmation, d'élection en élection, de la sur-représentation du vote FN dans des espaces aux caractéristiques territoriales convergentes milite pour une approche géopolitique. Mais celle-ci doit rester ouverte, ainsi que nous avons tenté de le montrer. La géopolitique du FN doit en effet intégrer, dans ses zones de force, une diversité d'intensité, d'une part, et de logique de report au second tour, d'autre part. Elle doit aussi tenir compte, dans les bureaux de vote populaires où ce parti est plus faible, du rôle des appareils municipaux, de l'encadrement militant, de la vie politique locale liée à la succession d'élections.

Surtout, l'analyse du vote FN devrait laisser de côté l'idée qu'il ne s'agirait que d'une vague protestation, et non d'un vote de conviction, laissant penser qu'il conviendrait, tout en le désapprouvant, de le comprendre avec toute la condescendance que cela implique. Le vote FN est un vote de conviction sous-tendu par la mise en exergue d'un bouc émissaire. Il l'est d'autant plus que ce vote se cristallise dans des territoires où, dans les années 1980, il apparaissait comme un surgissement provocateur, mais où, aujourd'hui, il est de plus en plus revendiqué, à défaut d'être le support d'un véritable appareil militant, ou la manifestation d'une véritable adhésion. La mise en évidence des causes (l'intériorisation d'un déclassement, d'une perte symbolique ou matérielle) n'est ainsi qu'une partie du travail d'analyse.

\section{Références citées :}

Alliès, P., Baraize, F., Négrier, E., 2003, « Une recomposition incertaine. Les élections présidentielles et législatives de 2002 en Languedoc-Roussillon », Pôle Sud n¹7, pp.101118

Braconnier, C., 2011, Une autre sociologie du vote. Les électeurs dans leurs contextes : bilan critique et perspectives, Cergy-Pontoise : LEJEP

Bussi, M. Colange, C. et Rivière, J. 2011. « Distance(s) à la ville et comportements électoraux. Quelques éclairages quantitatifs lors des derniers scrutins présidentiels », in D.Pumain et R.Mattéi (dir), Données urbaines 6, Paris : Anthropos, p. 33-42.

Dezé, A., 2012, Le Front National : à la conquête du pouvoir ? Paris : Armand Colin Girard, V., 2012, « Les votes à droite en périurbain : «frustration sociale des ménages modestes, ou recomposition des classes populaires ?, Métropolitiques, Avril 2012 Guilluy, C. 2010. Fractures françaises, Paris : Bourin Éditeur Huard, J., 2008, La conquête républicaine dans le Gard. Les moments, les idées, les hommes (1983-1848), Uzès : Inclinaison éditeurs

Le Bras, H., 1986, Les trois France, Paris : Odile Jacob (nouvelle édition 1996)

Lefebvre R., Sawicki F., 2006, La Société des socialistes, Bellecombe-en-Bauges, Editions du Croquant

Lehingue, P. 2003. « L'objectivation statistique des électorats : que savons-nous des électeurs du FN ? », in J.Lagroye (dir.), La politisation, Paris : Belin, p. 247-278 
Lehingue, P. 2011. Le vote. Approches sociologiques de l'institution et des comportements électoraux, Paris : la Découverte.

Martin, P., 2000, Comprendre les évolutions électorales, Paris : Presses de Science Po Mayer, N., 1987, « De Passy à Barbès : deux visages du vote Le Pen à Paris », Revue Française de Science Politique Vol.37 nº, p.891-906

Mayer, N., 2002, « Les hauts et les bas du vote Le Pen 2002, Revue Française de Science Politique Vol.52 n5-6, p.505-520

Négrier, E., 2001 (dir.) Les maîtres du sud. Géopolitique du Languedoc-Roussillon Golias Editeur

Négrier, E., Jourda, M., Ratinaud, S., 2011, « Guerre des roses en Languedoc. Les élections régionales de mars 2010 en Languedoc-Roussillon », Pôle Sud n³4, p.61-89

Noiriel, G., 2010, Le massacre des italiens, Aigues-Mortes 17 août 1893, Paris : Fayard Perrineau, P., 1988, « Le FN : l'écho politique de l'anomie urbaine », Esprit, mars-avril, p.22-38

Pôle Sud, 1998, revue, Élections et politiques régionales $\mathrm{n}^{\circ} 8$ - http://pole-sud.etud.univmontp1.fr/

Pôle Sud, 2008, revue, L'Europe du sud au urnes, n²9 - http://pole-sud.etud.univmontp1.fr/

Terra Nova, 2011, Gauche : quelle majorité électorale pour 2012 ?, 88 pages http://www.tnova.fr/essai/gauche-quelle-majorit-lectorale-pour-2012 